\title{
The expression of human tumor necrosis factor receptor (HTNFR) in the $\mathrm{CHO}$ cell line
}

\begin{abstract}
Tumor necrosis factor (TNF) is a multifunctional Proinflammatory cytokine that stimulates acute phase reactions by acting on TNFR1 and TNFR2 receptors. Deregulation of TNF production can lead to various uncontrolled inflammation diseases. One of the medical applications of TNF receptors (TNFR) is the treatment of inflammatory diseases such as rheumatoid arthritis (RA). The aim of the present research was to express TNFR1 in the eukaryotic system. Synthesized gene encoding the TNFR1 into $\mathrm{pGH}$ vector was sub cloned into the pEGFP-N1 plasmid and transfected into the CHO-K1 cell line. Purification of the recombinant TNFR was carried out by affinity chromatography. Green fluorescence in the cell culture confirmed the expressed protein and it was shown a sharp band in the western blot analysis. So CHO-K1 is able to express recombinant TNFR1 properly in lab scale conditions.
\end{abstract}

Keywords: purification, TNFR, pegfp-n1, affinity chromatography

\author{
Volume 2 Issue 3 - 2017
}

\author{
Shahla Korani,' Maryam Kaszadeh, ${ }^{2}$ Fatemeh \\ Yarian,' Mojgan Bandehpour ${ }^{1,2}$ \\ 'Department of Biotechnology, Shahid Beheshti University of \\ Medical Sciences, Tehran, Iran \\ ${ }^{2}$ Cellular and Molecular Biology Research Center, Shahid \\ Beheshti University of Medical Sciences, Iran \\ ${ }^{3}$ Department of Microbiology, Islamic Azad University, Tehran, \\ Iran
}

Correspondence: Mojgan Bandehpour, Department of Biotechnology, Shahid Beheshti University of Medical Sciences, Iran, Email bandehpour@gmail.com

Received: March 14, 2017 | Published: May 03, 2017

\section{Introduction}

Cytokines, including necrosis factor, play an important role in inducing and limiting inflammation. Cytokines play a pivotal role in coordinating the negative and positive signals required to produce and shape inflammatory responses. TNF and its receptors act as a well-known Proinflammatory cytokine system and they are important in various biological processes. ${ }^{1-3}$ The tumor necrosis factor receptor superfamily (TNFRSF) is named based on the first member of this family, that is, tumor necrosis factor (TNF), and it already has more than 40members with structural similarities. TNF leads to cytolysis of many tumor cells in vitro and has been found to cause hemorrhagic necrosis of transplanted tumors in rats, enhancing phagocytosis and the cytotoxicity of polymorpha nuclear neutrophils, as well as modulating the expression of many proteins, including lipoprotein lipase, class I antigens associated with major histo compatibility complex (MHC), and cytokines such as interleukin-1 and interleukin- $6 .{ }^{4} \mathrm{TNF}$ is a membrane-bound homodimer protein that can be released by proteases in the form of soluble cytokine. Both the membrane and the soluble cytokines are biologically active. It is produced by monocytes and macrophages, dendritic cells, B cells, T cells, and fibroblasts, as well as by fat cells, keratinocytes, breast, and colon epithelial cells, osteoblasts, mast cells, and many other cell types ${ }^{3,5}$ (TNF acts through two distinct receptors. NFR1 (also designated as p55) and TNFR2 are also known as P75. In addition, both receptors can be cleaved at the cell surface and changed to a soluble form, and from here, they can serve as decoy receptors (i.e., no signaling receptors) for the TNF function $^{6,7}$ (The dysregulation of TNF production can lead to uncontrolled inflammation and various diseases such as RA, which is characterized by chronic inflammation and bone erosion. Autoantibodies, cytokines, and chemokine are possible RA triggers. TNF receptors are used as therapeutic agents for the inhibition of cytokines according to their characteristics (targeting certain cytokines), high affinity (an affinity usually comparable to or greater than many existing anti-cytokine monoclonal antibodies), and lack of immunogenicity (unlike using rat mAbs used to treat humans, these receptors do not cause an antibody response in humans) ${ }^{8}$ (We hope that the recombinant TN-
F-alpha receptor will help in the treatment and control of inflammatory diseases. This study aims to investigate TNFR gene cloning and the expression of recombinant protein in eukaryotic cells, as well as purifying TNFR to be used in future research for the treatment of inflammatory diseases.

\section{Materials and methods}

\section{I-Synthesis of the TNFR gene in pGH vector}

The TNF receptor gene was selected based on the sequences in the Gen Bank (Gene ID: 7133). It was synthesized into pGH plasmid (Bioneer, Korea). Cloning accuracy was confirmed using a polymerase chain reaction (PCR). ${ }^{9}$ The primers designed to amplify the TNFR gene are as follows:

a. F: 5' ATATATTGCTAGCATGGCGCCCGTCGCCGTCTGGG3'(Tm:79.7).

b. R: 5' ATATATTGGATCCACTGGGCTTCATCCCAGCATC 3'(Tm:71.4).

These primers have the sequences required to identify and cut with the BamHI and NheI enzymes (Fermentas, Lithuania).

\section{2-Subcloning of the TNFR gene in the eukaryotic PEG- FP-NI expression vector}

The pGH vector containing TNFR gene and the pEGFP_N1 expression vector were digested with BamHI (Fermentas, Lithuania) and NheI enzymes. After purification of TNFR gene and the digested pEGFP N1 vector from agarose gel using the gel purification kit (Bioneer, Korea), ligation was carried out with T4 DNA Ligase (Fermentas, Lithuania). The recombinant plasmids were confirmed by colony PCR and restriction enzyme analysis.

Transfection of the recombinant vector in the $\mathrm{CHO}$ cell line using a calcium chloride buffer. $\mathrm{CHO}$ cell line was cultured routinely in the DMEM culture medium containing $10 \%$ fetal calf serum (FCS), $100 \mathrm{U} / \mathrm{ml}$ penicillin, and $100 \mu \mathrm{g} / \mathrm{ml}$ streptomycin at $37^{\circ} \mathrm{C} 5 \% \mathrm{CO}_{2}$. 
The cells were separated by Trypsin 24 hours before transfection. When the cell density reached $60-70 \%$, transfection was carried out with the pEGFP_TNFR plasmid. Three hours before transfection, the cell culture medium was replaced with serum-free DMEM. Two solutions were prepared in two separate tubes: $1 \mathrm{x}$ HBS (solution 1) and a solution containing $9 \mu \mathrm{g}$ of DNA and calcium chloride (2M) (solution 2). Solution 2 was added to Solution 1 and the resulting solution was incubated at room temperature for 20 minutes and added gradually to the cell flask. Following incubation for 16hours, the culture medium was replaced with the neomycin DMEM. Then, 40hours later, TNF receptor gene expression was analyzed by fluorescent microscopy.

\section{3-SDS-PAGE and western blotting}

The quality of the soluble expressed TNFR protein was confirmed by SDS-PAGE and Western blotting. ${ }^{10}$ After dialysis and precipitation of secreted protein in the culture medium, it was subjected to SDS-PAGE on a $12 \%$ polyacrylamide gel. The samples were mixed with a $2 \mathrm{X}$ loading buffer (125mM Tris, $20 \%$ Glycerol, $4 \%$ SDS and $0.01 \%$ bromophenol blue at $\mathrm{pH} 6.8$ ). Gel staining was carried out with Coomassie brilliant blue R-250. For western blotting, the recombinant proteins were separated on a $12 \%$ polyacrylamide gel and were electrophoretically transferred to a nitrocellulose membrane (Whatman, UK). The transblotted membrane was blocked for $2 \mathrm{~h}$ at room temperature with a blocking solution $(3 \%(\mathrm{w} / \mathrm{v})$ skimmed milk in TBS (Tris-Buffered Saline) (Sigma, USA). The membrane was incubated for $2 \mathrm{~h}$ at room temperature with the anti-TNFR (Abcam, UK) 1/5000 dilution monoclonal antibody. After washing, the membrane was incubated for $2 \mathrm{~h}$ at room temperature with secondary alkaline phosphatase conjugated anti-mouse antibody (Abcam, UK). After three times washing for $5 \mathrm{~min}$ with TBST, the NBT/BCIP substrate solution (Roche, Germany) was used to visualize the immunoreactivity.

\section{4-Purification of TNFR using Ni-NTA His Tag affinity Chromatography}

The recombinant His-tagged TNFR protein was purified via affinity chromatography using Ni2+-NTA agarose resin (Novagen, USA). After dialysis of cell culture medium with equilibration buffer $(20 \mathrm{mM}$ $\mathrm{NaH}_{2} \mathrm{PO} 4$, and $500 \mathrm{mM} \mathrm{NaCl}$ at $\mathrm{pH} 8.0$ ) it was loaded on an equilibrated resin. Three times washing of the resin was performed with $10 \mathrm{ml}$ of wash buffer $(10 \mathrm{mM}, 20 \mathrm{mM}, 30 \mathrm{mM}$ and $40 \mathrm{mM}$ gradient of imidazole, $20 \mathrm{mM} \mathrm{NaH}_{2} \mathrm{PO}_{4}$ and $500 \mathrm{mM} \mathrm{NaCl}$ at $\mathrm{pH} 8.0$ ), the bounded protein were eluted with $1 \mathrm{ml}$ of elution buffer $(300 \mathrm{mM}$ imidazole, $20 \mathrm{mM} \mathrm{NaH} \mathrm{PO}_{2}$ and $500 \mathrm{mM} \mathrm{NaCl}$ at $\left.\mathrm{pH} 8.0\right) .{ }^{11}$ Eluted protein was dialyzed in PBS at room temperature for $2 \mathrm{~h}$.
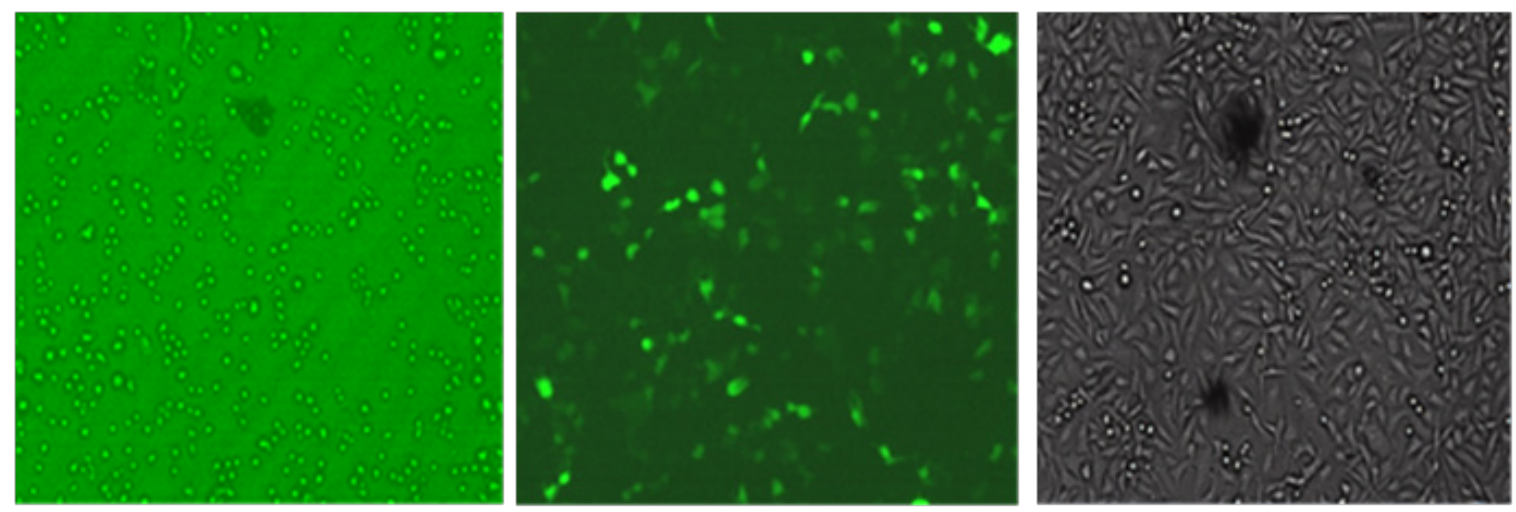

Figure 3 Micrograph of $\mathrm{CHO}$ cell line transfected with pEGFP-NI and recombinant pEGFP-NI-TNFR Left, CHO cells containing the pEGFP_NI plasmid as control, Right, CHO cells containing the recombinant plasmid (pEGFP-TNFR) with green fluorescent, $\mathrm{CHO}$ cells containing the recombinant plasmid ( $\mathrm{PEGFP}$ TNFR). 


\section{Discussion and conclusion}

In the present study, a eukaryotic system was used to express the recombinant protein of the TNF-alpha receptor. For this purpose, approved procedures were performed to clone the gene encoding this receptor into the pEGFP-N1 expression vector, while the CHO eukaryotic cell line was used to express the recombinant protein. ${ }^{12}$ In addition, due to the high expression of this plasmid and the fact that it is fluorescent with more production and more comfortable tracking during the expression stage were possible. ${ }^{13}$ The reason for choosing this receptor was the vital role of the TNF cytokine as a powerful inflammatory cytokine that is considered a strong mediator in inflammation and immune response. This receptor's expression level is very important because its overexpression leads to autoimmune diseases such as Rheumatoid Arthritis and tissue damage. ${ }^{14}$ TNF acts via two receptors, namely, TNFR1 and TNFR2. TNFR2 is expressed by immune and endothelial cells. TNF $\alpha$ antagonists are already among the most commonly used methods for clinical treatment. ${ }^{3,15}$ As a therapeutic agent for inhibiting the activity of TNF $\alpha$, TNFR has several advantages over some of the medications that suppress the immune system. Our research findings showed that CHO-K1 and the pEGFP-N1 vector are the appropriate system for the production of recombinant TNFR as drug protein whereas according to the Joshua A. Bornhorst and Joseph J. Falke, the protein can accept a polyhistidine affinity tag, and up to $95 \%$ purities with a $90 \%$ recovery of tagged protein can be achieved in a single purification step by purifying the Ni-NTA column of highly expressed protein. ${ }^{16}$ Also some researches were performed on the coding regions of receptors (TNFR1 and TNFR2) were integrated with the mammalian expression vector pRK5. Lewis et al. applied the TSA 201 cell line as a sub cloned off the human embryonic kidney cell line to detect the expression of the TNFRs proteins, as well as the lipofectamine technique to insert the recombinant plasmid into TSA 201 cells. ${ }^{17}$

Due to the side effects of the drugs used to treat some diseases, investing in such medications and optimizing them will be of significant economic and scientific value. Therefore, the production of TNF $\alpha$ receptor in the $\mathrm{CHO}$ cell line and of E.coli bacteria to produce more of that receptor to bind and block TNF $\alpha$ and prevent the inflammatory response in diseases such as Rheumatoid Arthritis, as well as to provide pain relief, is of great importance.

One of the benefits of this study, and the main difference between it and other researches is the application of the pEGFP-N1 vector to clone this gene. A primary characteristic of this vector is its expressivity in the eukaryotic system and the fact that it has green fluorescent protein (GFP), whose presence in this vector made it easier to identify and track the vector in our expression cell. ${ }^{13,18}$ Other distinguishing aspects of the project include the use of $\mathrm{CHO}$ cells to express the $\mathrm{TNF} \alpha$ receptor gene.

Based on the aforementioned features and the experience gained over the past two decades, as well as World Health Organization safety tests, therapeutic proteins produced by this cell line have been confirmed without any problems, so that today, annual sales of products produced by $\mathrm{CHO}$ cells amount to more than $\$ 30$ billion worldwide. ${ }^{19}$ As noted above, one of the benefits of $\mathrm{CHO}$ cells is their ability to produce glycosylated proteins, and some of the benefits of protein glycation are as follows: - It increases the duration of the action of proteins. Sugar entering the protein causes a certain conformation of the protein, and this allows easier identification by cell surface agents and therefore facilitates protein entry into the cell. Different plasmids have been used for cloning the TNF receptor gene. Many of these either have improper expression or have the proper expression but are of the viral type. Due to the toxicity of viral plasmids, along with ethical aspects, the use of non-viral plasmids seems to be safe in treatments compared to virus transmission systems. Accordingly, in this study, a non-viral vector was selected. The results will help us achieve the objectives of protein testing on animals, in addition to mass production of these proteins in the Ian as medicines. This, in turn, will allow us to compare the effect of the recombinant protein with common medicines in which proteins are purified as a result of gene expression.

\section{Acknowledgements}

This work was performed in the Cellular \& Molecular Biology Research Center of Shahid Beheshti University of Medical Sciences. This article was extracted from Shahla Korani's Ph.D. thesis. This study was funded by the deputy of Shahid Beheshti University of Medical Sciences (grant number 5061) and consulted to the doing of the experiment with the ethics code IR.SBMU.RAM.REC.1394.82.

\section{Conflict of interest}

Author declares that there is no conflict of interest.

\section{References}

1. Hehlgans T, Pfeffer K. The intriguing biology of the tumour necrosis factor/tumour necrosis factor receptor superfamily: players, rules and the games. Immunology. 2005;115(1):1-20.

2. Probert L. TNF and its receptors in the CNS: The essential, the desirable and the deleterious effects. Neuroscience. 2015;302:2-22.

3. Al-Lamki RS, Mayadas TN. Relevance of TNF- $\alpha$ in the context of other inflammatory cytokines in the progression of diabetic nephropathy. Kidney Int. 2015;87(4):281-296.

4. Aggarwal BB. Signalling pathways of the TNF superfamily: a doubleedged sword. Nature reviews Immunology. 2003;3(1):745-756.

5. Gray PW, Barrett K, Chantry D, et al. Proceedings of the National Academy of Sciences of the United States of America. 1990;87:7380-7384.

6. Bemelmans MH, van Tits LJ, Buurman WA. Tumor necrosis factor: function, release and clearance. Crit Rev Immunol. 1996;16(1):1-11.

7. Nophar Y, Kemper O, Brakebusch C, et al. Soluble forms of tumor necrosis factor receptors (TNF-Rs). The cDNA for the type I TNF-R, cloned using amino acid sequence data of its soluble form, encodes both the cell surface and a soluble form of the receptor. EMBO J. 1990;9(10): 3269-3278.

8. Fernandez-Botran R. Critical reviews in clinical laboratory sciences. 1999;36:165-224.

9. Sambrook J, Maniatis T. Molecular cloning: A laboratory manual. 1989.

10. Laemmli UK. Cleavage of structural proteins during the assembly of the head of bacteriophage T4. Nature. 1970;227(5259):680-685.

11. Sankian M, Yousefi M, Pazouki N, et al. Biotechnology and applied biochemistry. 2007;47:185-189.

12. Farnia P, Ghanavi J, Bahrami A, et al. Increased production of soluble vascular endothelial growth factors receptor-1 in CHO-cell line by using new combination of chitosan-protein lipid nanoparticles. Int J Clin Exp Med. 2015;8(1):1526-1533.

13. Cheng Y, Zhang $\mathrm{H}$, Mei S, et al. Construction of recombinant pEGFPN1-hPer2 plasmid and its expression in osteosarcoma cells. Oncol lett. 2016;11(4):2768-2772. 
14. Pallinti V, Ganesan N, Rajashekar G. Role of tumor necrosis factor-alpha in rheumatoid arthritis: a review. International Journal of Rheumatic Diseases. 2007;10(4):270-274.

15. Garfield BE, Krahl T, Appel S, et al. Clinical immunology. 2005;116:101-107.

16. Gerchman SE, Graziano V, Ramakrishnan V. Expression of chicken linker histones in E. coli: sources of problems and methods for overcoming some of the difficulties. Protein Expr Purif. 1994;5(3):242-251.
17. Lewis M, Tartaglia LA, Lee A, et al. Proceedings of the National Academy of Sciences of the United States of America. 1991;88:2830-2834.

18. Bornhorst FJ. Purification of proteins using polyhistidine affinity tags. Methods Enzymol. 2000;326:245-254.

19. Echelard Y. Current opinion in biotechnology. 1996;7:536-540. 